\title{
Implantable Biomaterial Based on Click Chemistry for Targeting Small Molecules
}

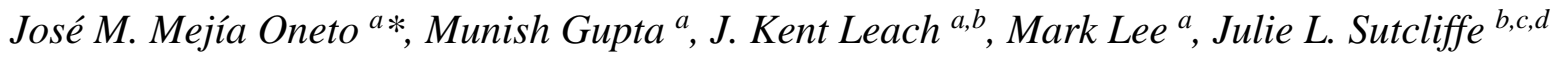

${ }^{a}$ Department of Orthopaedic Surgery, University of California, Davis, 4860 Y Street, Suite 3800, Sacramento, California 95817, USA.

${ }^{\mathrm{b}}$ Department of Biomedical Engineering, University of California, Davis, 451 Health Sciences Drive, Davis, California 95616, USA.

${ }^{c}$ Department of Internal Medicine, Division of Hematology/Oncology, University of California Davis, Sacramento, CA 95817, USA.

${ }^{\mathrm{d}}$ Center for Molecular and Genomic Imaging, University of California, Davis, 451 Health Sciences Drive, Davis, California 95616, USA.

* Corresponding Author. Tel.: +1 612353 7159; fax: +1 9167347904.

E-mail address: jose.mejiaoneto@ gmail.com (J.M. Mejía Oneto).

Keywords: alginate, hydrogel, click chemistry, drug delivery, in-vivo imaging, biodistribution

\begin{abstract}
Specific and targeted delivery of medical therapies continues to be a challenge for the optimal treatment of multiple medical conditions. Technological advances permit physicians to target most sites of the body. However, after the intervention, physicians rely on systemic medications that need frequent dosing and may have noxious side effects. A novel system combining the temporal flexibility of systemic drug delivery and the spatial control of injectable biomaterials would improve the spatiotemporal control of medical therapies. Here we present an implantable biomaterial that harnesses in-vivo click chemistry to enhance the delivery of suitable small molecules by an order of magnitude. The results demonstrate a simple and modular method to modify a biomaterial with small molecules in-vitro and present an example of a polysaccharide modified hours after in-vivo implantation. This approach provides the ability to precisely control
\end{abstract}


the moment when biochemical and/or physical signals may appear in an implanted biomaterial. This is the first step towards the construction of a biomaterial that enhances the spatial location of systemic small molecules via in-vivo chemical delivery.

\section{Introduction}

The specific and targeted delivery of therapies in post-operative pain management [1], localized antibiotics $[2,3]$ or chemotherapeutic delivery continues to be a challenge $[4,5]$. In addition, modern practitioners face the increasing incidence of antibiotic resistant pathogens [69], an aging patient population with multiple co-morbidities [10, 11], polypharmacy [12, 13], and higher use of prescription narcotics [14]. All of this means that there is a critical need for solutions that lead to medically efficient, cost-effective strategies for local therapeutic delivery with minimal side effects.

Modern drug delivery systems attempt to optimize the local and timely (spatial and temporal) delivery of therapeutics $[1,3,4,15]$. Existing biomaterials can serve as depots of medication through diffusion, affinity, immobilization or degradation [1]. However, after implantation most biomaterials cannot be modulated or modified and usually exhibit an initial burst of activity shortly after implantation $[1,16]$. These issues limit the application of biomaterials for multiple medical conditions that require the doses to be tailored at different time points $[16,17]$ or for which the most effective therapeutic agent is identified hours or days after implantation, e.g. after culture or pathology results are obtained.

Historically, cycloaddition reactions have been a powerful tool in the chemical synthesis of complex natural products [18]. One cycloaddition reaction, the inverse-electron demand Diels Alder reaction, has been used for several in-vivo click chemistry applications [19-21] and one of 
the most studied applications is for pretargeted tumor imaging [22-24]. Briefly, this technique involves the chemical conjugation of a monoclonal antibody (mAb) specific to a tumor marker with a trans-cyclooctene (TCO) moiety. The mAb-TCO conjugate is injected intravenously to a mouse growing a subcutaneous tumor. After an elapsed period of time, a chemical probe containing a tetrazine $(\mathrm{Tz})$ moiety with a handle for radiolabeling, for example the SPECT emitter, indium-111 chelated by DOTA $\left({ }^{111} \mathrm{In}-\mathrm{Tz} 2\right)$ is injected intravenously to the animal with subsequent localization of the radioactivity at the tumor. This demonstrates that $\mathrm{TCO}$ and $\mathrm{Tz}$ reagents are able to react with each other in-vivo at the surface of a tumor [22]. However, the need for mAb's and multi-day interventions hinder the application of this promising technology to other areas of medical research.

We envision that a new system combining the temporal flexibility of systemic drug delivery and the spatial control of injectable biomaterials could enhance the localization of small molecules (Fig. 1A). Here we report the design and construction of an alginate polymer (TCOgel 1) that was covalently modified to incorporate TCO molecules to its sugar backbone (Fig. 1B). Furthermore, we report the in-vitro and in-vivo interactions of this material with ${ }^{111} \mathrm{In}-\mathrm{Tz}-2$ molecules (Fig. 1B) [22]. The hypothesis is that TCO-gel 1 will react with circulating ${ }^{111} \mathrm{In}-\mathrm{Tz}-2$ molecules through an inverse electron demand Diels Alder reaction in a bioorthogonal fashion localizing the Tz molecules and their radioactive cargo to the TCO-gel $\mathbf{1 .}$

\section{Materials and methods}

\subsection{Materials}

All reagents and NMR solvents were purchased from Sigma-Aldrich (St. Louis, MO), unless otherwise noted. Chemical synthesis of $(R, E)-N$-(2-aminoethyl)-2-(cyclooct-4-en-1- 
yloxy)acetamide, precursor of TCO-Gel 1, was contracted to MBMR Biolabs Inc. (Brooklyn, NY). Silica gel was purchased from Silicycle (Quebec, Canada), while preparative TLC plates ( $20 \times 20 \mathrm{~cm} ; 1000 \mu \mathrm{m}$ in thickness) were purchased from Analtech (Newark, DE). Ultrapure (UP) MVG sodium alginate, a medium viscosity (>200 mPas) sodium alginate where a minimum of $60 \%$ of the monomer units are guluronate, was purchased from ProNova BioPharma ASA (Lysaker, Norway). [ $\left.{ }^{111} \mathrm{In}\right]$ Indium chloride solution was purchased from PerkinElmer (Waltham, US). Dulbecco's Phosphate Buffered Saline (DPBS, without calcium and magnesium) was obtained from Cellgro (Mediatech, Manassas, VA, USA) and had the following formulation (g/L): $\mathrm{KCl}, 0.20 ; \mathrm{KH}_{2} \mathrm{PO}_{4}, 0.20 ; \mathrm{NaCl}, 8.00 ; \mathrm{Na}_{2} \mathrm{HPO}_{4}$ (anhydrous), 1.15 .

\subsection{Syntheses of click chemistry components}

Detailed description of the synthesis of $[(R, E)-N$-(2-aminoethyl)-2-(cyclooct-4-en-1yloxy)acetamide], precursor of TCO-Gel 1, is provided in the Supplementary Information. Tz-2 was prepared according to published procedures [22]. The H-NMR spectra matched the one previously published and High-resolution Mass Spectrometry (HRMS) data were consistent with expected values. HRMS: $m / z[M+H]^{+}$calculated for $\mathrm{C}_{57} \mathrm{H}_{92} \mathrm{~N}_{13} \mathrm{O}_{20}$ 1278.6576, found 1278.6580 .

\subsection{Radiolabeling of $T z-2$}

Tz-2 was dissolved to a concentration of $1.0 \mathrm{mM}$ in $0.2 \mathrm{M}$ ammonium acetate (pH 7.0) and stored at $-80^{\circ} \mathrm{C}$ before use. An aliquot of Tz-2 was combined with a suitable amount of [ $\left.{ }^{111} \mathrm{In}\right]$ Indium chloride solution (approximately $700 \mathrm{kBq}$ of [ $\left.{ }^{111} \mathrm{In}\right]$ per nmole of Tz-2) and incubated for $10 \mathrm{~min}$ at $37^{\circ} \mathrm{C}$ under gentle agitation. Then, $5 \mu \mathrm{L}$ of $10 \mathrm{mM}$ DTPA was added and the solution was incubated for an additional 5 min. Typically, a quantitative labeling yield and a radiochemical purity greater than $97 \%$ were obtained; confirmed by radio-TLC and radioHPLC. For animal experiments, the solution of ${ }^{111} \mathrm{In}-\mathrm{Tz}-2$ was diluted with sterile saline. 


\subsection{Alginate chemical modification}

Each gram of UP MVG alginate was combined with $176 \mu$ moles of TCO-amine in standard carbodiimide chemistry conditions as previously described for RGD [25] and GHK [26] incorporation. Then the alginate product was purified by dialysis against deionized water containing decreasing salt concentrations for 4 days, frozen and lyophilized for 5-10 days until dry. A $2.5 \%$ alginate solution was obtained by adding DPBS, and alginate gels were fabricated by the addition of calcium. Covalent modification of alginate was confirmed through ${ }^{1} \mathrm{H}-\mathrm{NMR}$ studies (refer to Supplementary Information for spectra). The same protocol without the TCO addition was used for the construction of control gels. The in-vitro and in-vivo studies were done with TCO-Gel 1 from the exact same batch and used on the same day to minimize any variations in loading amount or loading efficiency.

For in-vitro experiments, $800 \mu 1$ of $2.5 \%$ alginate solution were mixed with $200 \mu 1$ of supersaturated $\mathrm{Ca}\left(\mathrm{SO}_{4}\right)_{2}$ solution $\left(0.21 \mathrm{~g} \mathrm{Ca}\left(\mathrm{SO}_{4}\right)_{2} / \mathrm{ml} \mathrm{ddH}_{2} \mathrm{O}\right)$. The solutions were mixed for $30 \mathrm{~s}$ using a three-way stopcock to achieve a final alginate concentration of $2 \%$. The mixture was allowed to gel between 2 glass plates in a custom plastic model and incubated for $20 \mathrm{~min}$ at room temperature. The entire volume had a uniform appearance consistent with gelation. The discs were picked up with a spatula and weighed individually. Typically, a premade disc weighed approximately $100 \mathrm{mg}$ and had roughly the following dimensions: $8 \mathrm{~mm}$ (diameter) and $2 \mathrm{~mm}$ (height).

For in-vivo use, the $2.5 \%$ alginate gel solution and the super-saturated calcium sulfate solution were mixed rapidly in the same proportions as mentioned above and immediately injected to the animal in the desired amount.

2.5 in-vitro quantification of TCO-gel 1 
The reactivity of control gel and TCO-Gel 1 discs were tested in $\mathrm{ddH}_{2} \mathrm{O}$. Using $2.0 \%$ (w/v) alginate solution in DPBS and calcium ions as crosslinkers, TCO-Gel 1 and control gel discs were created [25]. A premade disc (mean weight $0.10 \mathrm{~g}[0.06-0.12 \mathrm{~g}]$ ) was placed in a test tube and a solution containing ${ }^{111} \mathrm{In}-\mathrm{Tz}-2\left(1.04 \mathrm{MBq}, 0.47\right.$ nmoles in $0.20 \mathrm{~mL}$ of $\left.\mathrm{ddH}_{2} \mathrm{O}\right)$ added. Radioactivity levels were confirmed using a Capintec Dose Calibrator (Capintec, Inc.). The discs were then allowed to sit at room temperature for either ten $\min (n=1)$ or 14 hrs $(n=3)$. They were washed three times with $0.25 \mathrm{~mL}$ of $\mathrm{ddH}_{2} \mathrm{O}$ and the radioactivity was measured again.

\subsection{Animals and animal handling}

Female BALB/c mice (20-25 g body weight, Charles River Laboratories) were used for these studies. All animals were handled in accordance with a protocol approved by the University of California, Davis, Institutional Animal Care and Use Committee and experiments were performed according to the Principles of Laboratory Animal Care (NIH publication 85-23, revised 1985).

\section{7 in-vivo biodistribution study}

Either control gel or TCO-Gel 1 was injected subcutaneously at each flank area of the subject (mean weight $0.22 \mathrm{~g}[0.10-0.29 \mathrm{~g}]$ ). Approximately $3-4$ hours after gel injection, ${ }^{111} \mathrm{In}$ Tz-2 dissolved in $0.9 \% \mathrm{NaCl}(50 \mu \mathrm{L}$, mean dose $1.63 \mathrm{MBq}[1.45-1.79 \mathrm{MBq}]$, mean 0.88 nmoles $[0.78-1.01$ nmoles]) was injected intravenously (i.v) via a catheter into the tail vein of the mouse. At selected time points $(1,4,24,48 \mathrm{hrs})$ mice ( $\mathrm{n}=3 /$ time point) were euthanized, dissected and tissues of interest, such as blood, gallbladder, liver, heart, kidneys, pancreas, spleen, lungs, stomach, small intestine, large intestine, bladder, skin, muscle, bone, and brain were harvested as well as the gels and surrounding tissues (control gel, TCO-Gel 1). Solid tissues were washed with de-ionized water to remove excess blood and all collected material was 
weighed. The radioactivity associated with each sample was measured using a Wallac 1470 Wizard gamma counter (PerkinElmer, Inc.). Radioactivity uptake (decay corrected) is presented as percent injected dose per gram $(\% \mathrm{ID} / \mathrm{g})$. The results are expressed as mean $\pm \mathrm{SD}$.

\subsection{SPECT/CT imaging study}

Either control gel or TCO-Gel 1 was injected subcutaneously at each flank area of the subject ( $0.23 \mathrm{~g}$ for control gel and $0.17 \mathrm{~g}$ for TCO-Gel 1). Approximately $3 \mathrm{hrs}$ after injection of the gel, ${ }^{111} \mathrm{In}-\mathrm{Tz}-2$ dissolved in $0.9 \% \mathrm{NaCl}(90 \mu \mathrm{L}, 38.8 \mathrm{MBq}, 21 \mathrm{nmoles})$ was injected i.v. via a catheter into the tail vein of the mouse. At 4, 24 and 48 hrs after ${ }^{111}$ In-Tz-2 injection, the mouse was anesthetized with 1-2\% isoflurane and positioned in the prone position on an animal bed equipped with a nose cone for anesthesia and tubing with warm water to maintain adequate body temperature. SPECT imaging was performed with a SPECT/CT Inveon Preclinical Imaging Station (Siemens Healthcare) for 60 min using a 5-pin hole collimator. Immediately after SPECT acquisition CT images were acquired. Coregistration of SPECT and CT images was performed using fiducial markers and the Inveon Research Workplace (IRW) software (Siemens Healthcare).

\subsection{Statistics}

Group variation is described as the mean \pm one standard deviation. For in-vitro and biodistribution studies, single groups were compared with a two-tailed paired $t$-test. Groups with $p<0.05$ were considered significantly different. Microsoft Excel version 12.8.9 was used for graphs and statistical calculations.

\section{Results}

\subsection{Fabrication and in-vitro characterization of click chemistry-modified biomaterial}


The TCO-Gel 1 discs maintained significantly more activity than the control gels (Fig. 2). The level of activity of the control gel was equivalent to $0.86 \pm 0.21$ nmoles/g of ${ }^{111} \mathrm{In}-\mathrm{Tz} 2$ $(\mathrm{n}=3,14$ hours), while the amount of radioactive molecules bound covalently to TCO-Gel $\mathbf{1}$ was $2.94 \pm 0.05$ nmoles/g after 14 hours of incubation $(n=3)$. Comparison between the control and the TCO-gel 1 revealed a statistical significant difference $(p$-value $<0.05)$ as measured by paired $t$-test. These results confirmed that this concept was viable in a test tube.

In addition, a single sample was stopped after just 10 minutes. The level of activity of the control gel was equivalent to 0.75 nmoles/g of ${ }^{111} \mathrm{In}-\mathrm{Tz} 2(\mathrm{n}=1)$. On the other hand, the amount of radioactivity at TCO-gel 1 at 10 minutes was $1.03 \mathrm{nmole} / \mathrm{g}(\mathrm{n}=1)$.

\section{2 in-vivo characterization of local delivery}

To assess the in-vivo relevance of the observed in-vitro binding, Balb/c mice were injected at the flank area with subcutaneous injections of either control gel or TCO-Gel 1. After approximately 3 hours the mice received a tail-vein i.v. injection of ${ }^{111} \mathrm{In}-\mathrm{Tz} 2$. The levels of radioactivity delivered to each organ or hydrogel site were determined by biodistribution studies at 1, 4, 24 and 48 hours with 3 mice per time point (Fig. 3). The mean weight of gel retrieved was $0.15 \mathrm{~g} \pm 0.04 \mathrm{~g}$ ( $69 \%$ of injected gel). In order to obtain an estimate of the remaining $30 \%$ of gel injected, we carefully collected the tissue surrounding each gel $(0.12 \mathrm{~g} \pm 0.04 \mathrm{~g})$. In addition, SPECT images of one mouse were taken at 4, 24 and 48 hours to visualize the spatial distribution of indium-111 (Fig. 4).

Our results indicate that we achieved an in-vivo click reaction with delivery of greater than $4 \%$ intravenous ID/g in the subcutaneous space of a murine model at 1 hour. In contrast, the radioactivity level delivered to unmodified muscle or skin tissues was $<0.3 \% \mathrm{ID} / \mathrm{g}$. The level of radioactivity at the control gel and surrounding tissue was $<1.8 \% \mathrm{ID} / \mathrm{g}$ at 1 hour and 
declined rapidly to background levels afterwards $(<0.4 \% \mathrm{ID} / \mathrm{g})$. In contrast, the amount of radioactivity at the TCO-gel 1 site was maintained to a level above $1 \% \mathrm{ID} / \mathrm{g}$ even after 48 hours. The difference between the groups was statistically significant at all time points (Fig. 3A and 4). Our results are also consistent with previous literature reports, that indicate ${ }^{111} \mathrm{In}-\mathrm{Tz} \mathbf{2}$ is excreted renally and has a short in-vivo half-life of about 10 minutes [22]. The major activity, other than TCO-gel 1, was found at the kidneys (2.3\% ID/g) and the bladder (1.1\% ID/g) at 1 hour. The level of activity circulating in the blood at 1 hour was comparable to the level of the control gel $(1.7 \% \mathrm{ID} / \mathrm{g})$. The activity at the lungs was $0.7 \% \mathrm{ID} / \mathrm{g}$ at 1 hour and declined rapidly. All other organs examined were $<0.5 \% \mathrm{ID} / \mathrm{g}$ at 1 hour and then declined to background levels. Also, ${ }^{111}$ In-Tz 2 appeared unable to cross the blood brain barrier and the brain had an activity level of $<0.1 \% \mathrm{ID} / \mathrm{g}$ at 1 hour.

\section{Discussion}

Since its emergence in 2001, click chemistry [19, 27, 28] has been applied to many fields of modern chemical science. One of its most recent incarnations is for in-vivo pretargeted nuclear imaging approaches. The technique entails the delivery of a molecular load through antibodies to a subcutaneous tumor $[20,22,23]$. Tumors, well known to have leaky vasculature and increased blood flow $[1,29]$, likely maximize the exposure of the mAb-TCO to the Tz radioprobe. Moreover, while a $\mathrm{mAb}$ can provide exquisite localization, the system has some limitations. Antibodies require: 1) an identifiable and accessible target; 2) specificity to said target; 3$)$ the ability to be chemically modified to carry a TCO cargo and maintain specificity $[5$, 22]; and 4) rapid clearance from the blood stream of unbound mAb's to minimize unwanted side 
effects [24]. The platform we describe in this report circumvents the need for mAb's, providing a modular and flexible platform for in-vivo small molecule delivery.

Previous methods to assess the incorporation of small molecules to an alginate polymer use radiolabeled molecules as tracers and a comparison of the activity between the product and the initial reaction solution [25]. However, the trans chemical bond of TCO is known isomerize to the less reactive cis-bond under a number of conditions [30]. Since the purification of the alginate polymer after the amidation reaction involves multi-day dialysis and a lyophilization step, it is unlikely that the initial incorporation of TCO to the alginate is representative of the number of TCO molecules that resisted isomerization during the reaction conditions needed to obtain TCO-Gel 1. Thus we developed an in-vitro protocol to quantify the functional loading of TCO molecules in TCO-Gel 1.

Careful evaluation of the in-vitro results reveals that a small amount of ${ }^{111} \mathrm{In}-\mathrm{Tz} 2$ binds the control gel and does not seem to increase over time. The most likely explanation is that nonspecific forces between ${ }^{111} \mathrm{In}-\mathrm{Tz}-2$ and the polysaccharide backbone of alginate lead to this finding. The affinity of cationic small molecules to alginate has been well characterized in the literature [1]. In contrast, the amount of radioactivity bound to TCO-gel 1 seems to increase over time, and after 14 hours, there is a statistical significant difference in the amount of ${ }^{111} \mathrm{In}-\mathrm{Tz}-2$ bound between TCO-gel 1 and control gel. This encouraging piece of data led us to test our platform in an animal model.

Our results show that ${ }^{111} \mathrm{In}-\mathrm{Tz}-2$, a molecule with expedient renal clearance and an invivo half-life of ten minutes can be captured by our platform and its local concentration can be increased by an order of magnitude $(0.3 \% \mathrm{ID} / \mathrm{g}$ to $4.0 \% \mathrm{ID} / \mathrm{g})$. Consistent with our in-vitro studies, there is a small level of radioactivity present at the control gel, which seems to correlate 
with blood radioactivity levels and decrease rapidly as one would expect for non-specific binding forces. The level of radioactivity at the experimental site of TCO-gel $\mathbf{1}$ at 1 hour is significantly higher and is maintained above background for two days. The results of the imaging and biodistribution studies support the enhanced localization of systemic ${ }^{111} \mathrm{In}-\mathrm{Tz}-2$ to TCO-gel 1.

Our results have elucidated the following: (1) Alginate is a suitable polymer backbone to carry out in-vivo chemistry; (2) The perfusion of a biomaterial in healthy subcutaneous space allows the local delivery of a compound $\left({ }^{111} \mathrm{In}-\mathrm{Tz} 2\right)$ even with an in-vivo half-life of 10 minutes [22]; and (3) TCO-gel 1 can be implanted for hours and be an adequate partner for in-vivo chemistry. Nonetheless, the current proof of concept also contains several limitations. The small molecules delivered remain covalently attached to the macromolecular delivery device (TCO-gel 1).

While the vast majority of therapeutic agents work in solution, there are several reports of the retained in-vivo activity of small molecules and proteins after covalent linkage to biomaterials. For instance, it has been shown the covalent modification with vancomycin of titanium alloys [31-35] and bone grafts [36-39] inhibit bacterial biofilm formation in-vitro and in-vivo $[2,40]$. Others have reported the benefits of antimicrobial peptides that are covalently immobilized onto biomaterial surfaces [41]. Furthermore, multiple studies have shown that signaling proteins such as vascular endothelial growth factor [42], granulocyte macrophage colony stimulating factor [43], interferon-gamma [44], among others, can be successfully immobilized and maintain their activity. These studies illustrate the benefits of biomaterials that are covalently modified with therapeutic agents. The common denominator of these strategies is that the therapeutic agents have extracellular targets. Further studies are needed to expand the 
use of the platform presented to therapeutic agents with intracellular targets and are currently underway.

In addition, further studies are required to determine the in-vivo degradation pattern of TCO-gel 1. Alginate hydrogel is a biocompatible and cytocompatible material that has found wide use in biomedical applications including the delivery of inductive molecules $[25,26]$, the transplantation of cells into defect sites [1] and even as devices for myocardial regeneration after heart attacks in humans $[45,46]$. Furthermore, alginate hydrogel can be modified to undergo breakdown or resist degradation [1], thus tailoring how long it stays in the body [47]. As these are the initial studies on TCO-gel, there are no previous literature reports for the degradation or activity of this specific modified biomaterial. In the course of our animal studies, no untoward effects were noted in the mice that had the gel implanted for a week (unpublished studies). Further studies are needed to determine whether small molecules such as Tz or TCO are released from the hydrogel (e.g., by hydrolysis of the amide bond) and whether this causes any negative reactions.

Other avenues for exploration of this platform include the study of the reaction rate of TCO-Gel $\mathbf{1}$ as well as the nature of the increase in binding with higher amounts of reactive molecules. Moreover, published work indicates that chemical optimizations can allow TCO molecules attached to antibodies to remain active in-vivo for up to 3 days [30], but further studies are needed to determine how long after implantation TCO-gel $\mathbf{1}$ is a viable in-vivo chemistry reaction partner.

\section{Conclusions}


This investigation supports our hypothesis that an implantable scaffold can be chemically modulated through principles of click chemistry and increase the concentration of systemic small molecule by an order of magnitude in the subcutaneous space. This is a novel concept for the control of local delivery of a molecular payload through a macromolecular device and presents proof for the spatiotemporal chemical modification of biomaterials after implantation by targeted delivery of systemic small molecules.

\section{Acknowledgements}

We thank H. Reddi and M. Royzen for helpful discussions, M. Wilson for help during the statistical analysis of preliminary data, Z. Al-Rashid for bringing to the attention of J.M.M.O the work on TCO-based bioorthogonal reactions, M. Hughbanks for initial assistance with gel synthesis, L. Knight and N. Bauer for initial help on radiochemistry and animal studies. We also thank the CMGI staff for excellent technical help during radiochemistry and imaging studies, the UC Davis NMR facility for technical assistance with NMR data collection of hydrogels and the Hammock group for access to a FTIR spectrometer.

Author Contributions: J.M.M.O. contributed with conception, experimental design, grant writing, manuscript writing, and was physically involved in all experiments. M.G \& M.L. contributed equally with mentorship through monthly meetings. J.K.L. provided physical, intellectual support and advice on hydrogel studies. J.L.S. provided physical, intellectual support and advice during preliminary radiochemistry and preliminary animal studies. Contributing author names are in alphabetical order.

Financial Disclosure: J.M.M.O was supported by the Denny and Jeanene Dickenson Resident Research Fellowship of the Department of Orthopaedic Surgery at the University of California, Davis. Support for this research was provided by the University of California, Davis Orthopaedic Surgery Department, the Office of Science, United States Department of Energy, DE-SC0008385, a grant from the Orthopaedic Trauma Association (OTA) and a Resident Clinician Scientist Training Grant from the Orthopaedic Research \& Educational Fund (OREF). 


\section{References}

[1] Kearney CJ, Mooney DJ. Macroscale delivery systems for molecular and cellular payloads. Nat Mater. 2013;12:1004-17.

[2] Hickok NJ, Shapiro IM. Immobilized antibiotics to prevent orthopaedic implant infections. Adv Drug Deliv Rev. 2012;64:1165-76.

[3] Velema WA, van der Berg JP, Hansen MJ, Szymanski W, Driessen AJM, Feringa BL. Optical control of antibacterial activity. Nat Chem. 2013;5:924-28.

[4] Lee JH, Chen KJ, Noh SH, Garcia MA, Wang H, Lin WY, et al. On-demand drug release system for in vivo cancer treatment through self-assembled magnetic nanoparticles. Angew Chem Int Ed Engl. 2013;52:4384-8.

[5] Davis ME, Chen ZG, Shin DM. Nanoparticle therapeutics: an emerging treatment modality for cancer. Nat Rev Drug Discov. 2008;7:771-82.

[6] Piddock LJ. The crisis of no new antibiotics--what is the way forward? Lancet Infect Dis. 2012;12:249-53.

[7] Enright MC, Robinson DA, Randle G, Feil EJ, Grundmann H, Spratt BG. The evolutionary history of methicillin-resistant Staphylococcus aureus (MRSA). Proc Natl Acad Sci U S A. 2002;99:7687-92.

[8] Bartlett JG, Gilbert DN, Spellberg B. Seven Ways to Preserve the Miracle of Antibiotics. Clin Infect Dis. 2013;56:1445-50.

[9] Antimicrobial resistance: global report on surveillance 2014. Geneva, World Health Organization, 2014.

(http://www.who.int/iris/bitstream/10665/112642/1/9789241564748_eng.pdf, accessed 5 May 2014).

[10] Barnett K, Mercer SW, Norbury M, Watt G, Wyke S, Guthrie B. Epidemiology of multimorbidity and implications for health care, research, and medical education: a crosssectional study. Lancet. 2012;380:37-43.

[11] Parekh AK, Barton MB. The challenge of multiple comorbidity for the US health care system. JAMA. 2010;303:1303-4.

[12] Boyd CM, Darer J, Boult C, Fried LP, Boult L, Wu AW. Clinical practice guidelines and quality of care for older patients with multiple comorbid diseases: implications for pay for performance. JAMA. 2005;294:716-24.

[13] Gurwitz JH. Polypharmacy: a new paradigm for quality drug therapy in the elderly? Arch Intern Med. 2004;164:1957-9.

[14] Holman JE, Stoddard GJ, Higgins TF. Rates of prescription opiate use before and after injury in patients with orthopaedic trauma and the risk factors for prolonged opiate use. J Bone Joint Surg Am. 2013;95:1075-80.

[15] Laurencin CT, Khan Y. Regenerative engineering. Sci Transl Med. 2012;4:160ed9.

[16] Pashuck ET, Stevens MM. Designing regenerative biomaterial therapies for the clinic. Sci Transl Med. 2012;4:160sr4.

[17] Tibbitt MW, Anseth KS. Dynamic microenvironments: the fourth dimension. Sci Transl Med. 2012;4:160ps24.

[18] Mejia-Oneto JM, Padwa A. Application of the Rh(II) cyclization/cycloaddition cascade for the total synthesis of (+/-)-aspidophytine. Org Lett. 2006;8:3275-8.

[19] Kolb HC, Finn MG, Sharpless KB. Click Chemistry: Diverse Chemical Function from a Few Good Reactions. Angew Chem Int Ed Engl. 2001;40:2004-21. 
[20] Devaraj NK, Thurber GM, Keliher EJ, Marinelli B, Weissleder R. Reactive polymer enables efficient in vivo bioorthogonal chemistry. Proc Natl Acad Sci U S A. 2012;109:4762-7.

[21] Stairs S, Neves AA, Stockmann H, Wainman YA, Ireland-Zecchini H, Brindle KM, et al. Metabolic glycan imaging by isonitrile-tetrazine click chemistry. Chembiochem. 2013;14:10637.

[22] Rossin R, Verkerk PR, van den Bosch SM, Vulders RC, Verel I, Lub J, et al. In vivo chemistry for pretargeted tumor imaging in live mice. Angew Chem Int Ed Engl. 2010;49:33758.

[23] Zeglis BM, Sevak KK, Reiner T, Mohindra P, Carlin SD, Zanzonico P, et al. A pretargeted PET imaging strategy based on bioorthogonal Diels-Alder click chemistry. J Nucl Med.

2013;54:1389-96.

[24] Rossin R, Lappchen T, van den Bosch SM, Laforest R, Robillard MS. Diels-Alder reaction for tumor pretargeting: in vivo chemistry can boost tumor radiation dose compared with directly labeled antibody. J Nucl Med. 2013;54:1989-95.

[1] Kearney CJ, Mooney DJ. Macroscale delivery systems for molecular and cellular payloads. Nat Mater. 2013;12:1004-17.

[2] Hickok NJ, Shapiro IM. Immobilized antibiotics to prevent orthopaedic implant infections. Adv Drug Deliv Rev. 2012;64:1165-76.

[3] Velema WA, van der Berg JP, Hansen MJ, Szymanski W, Driessen AJM, Feringa BL. Optical control of antibacterial activity. Nat Chem. 2013;5:924-8.

[4] Lee JH, Chen KJ, Noh SH, Garcia MA, Wang H, Lin WY, et al. On-demand drug release system for in vivo cancer treatment through self-assembled magnetic nanoparticles. Angew Chem Int Ed Engl. 2013;52:4384-8.

[5] Davis ME, Chen ZG, Shin DM. Nanoparticle therapeutics: an emerging treatment modality for cancer. Nat Rev Drug Discov. 2008;7:771-82.

[6] Piddock LJ. The crisis of no new antibiotics--what is the way forward? Lancet Infect Dis. 2012;12:249-53.

[7] Enright MC, Robinson DA, Randle G, Feil EJ, Grundmann H, Spratt BG. The evolutionary history of methicillin-resistant Staphylococcus aureus (MRSA). Proc Natl Acad Sci U S A. 2002;99:7687-92.

[8] Bartlett JG, Gilbert DN, Spellberg B. Seven Ways to Preserve the Miracle of Antibiotics. Clin Infect Dis. 2013;56:1445-50.

[9] Organization WH. Antimicrobial resistance: global report on surveillance 2014. 2014.

[10] Barnett K, Mercer SW, Norbury M, Watt G, Wyke S, Guthrie B. Epidemiology of multimorbidity and implications for health care, research, and medical education: a crosssectional study. Lancet. 2012;380:37-43.

[11] Parekh AK, Barton MB. The challenge of multiple comorbidity for the US health care system. JAMA. 2010;303:1303-4.

[12] Boyd CM, Darer J, Boult C, Fried LP, Boult L, Wu AW. Clinical practice guidelines and quality of care for older patients with multiple comorbid diseases: implications for pay for performance. JAMA. 2005;294:716-24.

[13] Gurwitz JH. Polypharmacy: a new paradigm for quality drug therapy in the elderly? Arch Intern Med. 2004;164:1957-9.

[14] Holman JE, Stoddard GJ, Higgins TF. Rates of prescription opiate use before and after injury in patients with orthopaedic trauma and the risk factors for prolonged opiate use. J Bone Joint Surg Am. 2013;95:1075-80. 
[15] Laurencin CT, Khan Y. Regenerative engineering. Sci Transl Med. 2012;4:160ed9.

[16] Pashuck ET, Stevens MM. Designing regenerative biomaterial therapies for the clinic. Sci Transl Med. 2012;4:160sr4.

[17] Tibbitt MW, Anseth KS. Dynamic microenvironments: the fourth dimension. Sci Transl Med. 2012;4:160ps24.

[18] Mejia-Oneto JM, Padwa A. Application of the Rh(II) cyclization/cycloaddition cascade for the total synthesis of (+/-)-aspidophytine. Org Lett. 2006;8:3275-8.

[19] Kolb HC, Finn MG, Sharpless KB. Click Chemistry: Diverse Chemical Function from a Few Good Reactions. Angew Chem Int Ed Engl. 2001;40:2004-21.

[20] Devaraj NK, Thurber GM, Keliher EJ, Marinelli B, Weissleder R. Reactive polymer enables efficient in vivo bioorthogonal chemistry. Proc Natl Acad Sci U S A. 2012;109:4762-7.

[21] Stairs S, Neves AA, Stockmann H, Wainman YA, Ireland-Zecchini H, Brindle KM, et al. Metabolic glycan imaging by isonitrile-tetrazine click chemistry. Chembiochem. 2013;14:10637.

[22] Rossin R, Verkerk PR, van den Bosch SM, Vulders RC, Verel I, Lub J, et al. In vivo chemistry for pretargeted tumor imaging in live mice. Angew Chem Int Ed Engl. 2010;49:33758 .

[23] Zeglis BM, Sevak KK, Reiner T, Mohindra P, Carlin SD, Zanzonico P, et al. A pretargeted PET imaging strategy based on bioorthogonal Diels-Alder click chemistry. J Nucl Med. 2013;54:1389-96.

[24] Rossin R, Lappchen T, van den Bosch SM, Laforest R, Robillard MS. Diels-Alder reaction for tumor pretargeting: in vivo chemistry can boost tumor radiation dose compared with directly labeled antibody. J Nucl Med. 2013;54:1989-95.

[25] Rowley JA, Madlambayan G, Mooney DJ. Alginate hydrogels as synthetic extracellular matrix materials. Biomaterials. 1999;20:45-53.

[26] Jose S, Hughbanks ML, Binder BY, Ingavle GC, Leach JK. Enhanced trophic factor secretion by mesenchymal stem/stromal cells with Glycine-Histidine-Lysine (GHK)-modified alginate hydrogels. Acta Biomater. 2014;10:1955-64.

[27] Royzen M, Yap GP, Fox JM. A photochemical synthesis of functionalized transcyclooctenes driven by metal complexation. J Am Chem Soc. 2008;130:3760-1.

[28] Blackman ML, Royzen M, Fox JM. Tetrazine ligation: fast bioconjugation based on inverse-electron-demand Diels-Alder reactivity. J Am Chem Soc. 2008;130:13518-9.

[29] Jain RK. Normalization of tumor vasculature: an emerging concept in antiangiogenic therapy. Science. 2005;307:58-62.

[30] Rossin R, van den Bosch SM, Ten Hoeve W, Carvelli M, Versteegen RM, Lub J, et al. Highly reactive trans-cyclooctene tags with improved stability for Diels-Alder chemistry in living systems. Bioconjug Chem. 2013;24:1210-7.

[31] Jose B, Antoci V, Jr., Zeiger AR, Wickstrom E, Hickok NJ. Vancomycin covalently bonded to titanium beads kills Staphylococcus aureus. Chem Biol. 2005;12:1041-8.

[32] Antoci V, Jr., Adams CS, Hickok NJ, Shapiro IM, Parvizi J. Vancomycin bound to Ti rods reduces periprosthetic infection: preliminary study. Clin Orthop Relat Res. 2007;461:88-95. [33] Antoci V, Jr., Adams CS, Parvizi J, Ducheyne P, Shapiro IM, Hickok NJ. Covalently attached vancomycin provides a nanoscale antibacterial surface. Clin Orthop Relat Res. 2007;461:81-7. 
[34] Antoci V, Jr., King SB, Jose B, Parvizi J, Zeiger AR, Wickstrom E, et al. Vancomycin covalently bonded to titanium alloy prevents bacterial colonization. J Orthop Res. 2007;25:85866.

[35] Antoci V, Jr., Adams CS, Parvizi J, Davidson HM, Composto RJ, Freeman TA, et al. The inhibition of Staphylococcus epidermidis biofilm formation by vancomycin-modified titanium alloy and implications for the treatment of periprosthetic infection. Biomaterials. 2008;29:468490.

[36] Ketonis C, Adams CS, Barr S, Aiyer A, Shapiro IM, Parvizi J, et al. Antibiotic modification of native grafts: improving upon nature's scaffolds. Tissue Eng Part A. 2010;16:2041-9. [37] Ketonis C, Barr S, Adams CS, Hickok NJ, Parvizi J. Bacterial colonization of bone allografts: establishment and effects of antibiotics. Clin Orthop Relat Res. 2010;468:2113-21. [38] Ketonis C, Barr S, Adams CS, Shapiro IM, Parvizi J, Hickok NJ. Vancomycin bonded to bone grafts prevents bacterial colonization. Antimicrob Agents Chemother. 2011;55:487-94. [39] Ketonis C, Barr S, Shapiro IM, Parvizi J, Adams CS, Hickok NJ. Antibacterial activity of bone allografts: comparison of a new vancomycin-tethered allograft with allograft loaded with adsorbed vancomycin. Bone. 2011;48:631-8.

[40] Stewart S, Barr S, Engiles J, Hickok NJ, Shapiro IM, Richardson DW, et al. Vancomycinmodified implant surface inhibits biofilm formation and supports bone-healing in an infected osteotomy model in sheep: a proof-of-concept study. J Bone Joint Surg Am. 2012;94:1406-15. [41] Costa F, Carvalho IF, Montelaro RC, Gomes P, Martins MC. Covalent immobilization of antimicrobial peptides (AMPs) onto biomaterial surfaces. Acta Biomater. 2011;7:1431-40. [42] Rahman N, Purpura KA, Wylie RG, Zandstra PW, Shoichet MS. The use of vascular endothelial growth factor functionalized agarose to guide pluripotent stem cell aggregates toward blood progenitor cells. Biomaterials. 2010;31:8262-70.

[43] Poloso NJ, Nagarajan S, Mejia-Oneto JM, Selvaraj P. GPI-anchoring of GM-CSF results in active membrane-bound and partially shed cytokine. Mol Immunol. 2002;38:803-16.

[44] Leipzig ND, Wylie RG, Kim H, Shoichet MS. Differentiation of neural stem cells in threedimensional growth factor-immobilized chitosan hydrogel scaffolds. Biomaterials. 2011;32:5764.

[45] Lee LC, Wall ST, Klepach D, Ge L, Zhang Z, Lee RJ, et al. Algisyl-LVR with coronary artery bypass grafting reduces left ventricular wall stress and improves function in the failing human heart. Int J Cardiol. 2013;168:2022-8.

[46] Lee RJ, Hinson A, Helgerson S, Bauernschmitt R, Sabbah HN. Polymer-based restoration of left ventricular mechanics. Cell Transplant. 2013;22:529-33.

[47] Boontheekul T, Kong HJ, Mooney DJ. Controlling alginate gel degradation utilizing partial oxidation and bimodal molecular weight distribution. Biomaterials. 2005;26:2455-65. 


\section{Figure Captions}

Fig. 1. Experimental Description. a) The method consists of the initial injection of a solid carrier covalently attached to TCO molecules. This is followed by a systemic injection of small molecules synthetically coupled to tetrazine moieties. When the two entities are in close proximity, they react irreversibly attaching the therapy to the solid carrier and thus localizing the small molecule. b) Two molecular probes were utilized for the study: TCO-Gel 1 and ${ }^{111} \mathrm{In}-\mathrm{Tz}$ 2.

Fig. 2. Quantification of TCO-Gel 1 loading in-vitro. For in-vitro studies, pre-weighed gel discs (experimental or control) were mixed with a solution of ${ }^{111} \mathrm{In}-\mathrm{Tz}-2$ (1.04 MBq, 0.47 nmoles in $0.20 \mathrm{~mL}$ of ddH $\mathrm{H}_{2}$ ) for 10 min or 14 hours. During a 10-minute exposure a sample of TCO-Gel 1 (green bars) bonded $1.78 \mathrm{nmoles} / \mathrm{g}$, while the control gel (blue bars) maintained 0.75 nmoles $/ g$ of ${ }^{111} \mathrm{In}-\mathrm{Tz}-2$. At 14 hours the amount of ${ }^{111} \mathrm{In}-\mathrm{Tz}-2$ attached to control gel remained constant at $0.86 \mathrm{nmoles} / \mathrm{g}$. The TCO-Gel 1 bound $2.94 \mathrm{nmoles} / \mathrm{g}$. Error bars represent mean SD for $n=3$. * $p$-value $<0.05$ as measured by paired $t$-test.

Fig. 3. Biodistribution results of ${ }^{111} \mathrm{In}-\mathrm{Tz}-2$. a) Mice $(\mathrm{n}=3$ ) bearing subcutaneous TCO-Gel 1 (mean weight $0.23 \mathrm{~g}$ [0.10-0.29 g]) and control gel (Mean weight $0.23 \mathrm{~g}$ [0.16-0.25 g]) were administered ${ }^{111} \mathrm{In}-\mathrm{Tz} 2$ (mean $1.63 \mathrm{MBq}[1.45$ $-1.79 \mathrm{MBq}]$ ) via tail vein injection $(t=0)$. b) Mean difference at specific time points between TCO-Gel 1 (green bars) and control gel (blue bars). Bars indicate \%ID/g per organ at 1, 4, 24 and 48 hrs. Data are mean $\pm S D ; n=3$. ${ }^{*} p$ value $<0.05$ as measured by paired $t$-test.

Fig. 4. SPECT Imaging of ${ }^{111} \mathrm{In}-\mathrm{Tz}-2$ biodistribution. ${ }^{111} \mathrm{In}-\mathrm{Tz}-2(90 \mu \mathrm{L}, 38.8 \mathrm{MBq}, 21 \mathrm{nmoles})$ was systemically administered to a mouse via tail vein injection 3 hours after subcutaneous injection of TCO-Gel 1 ( $0.17 \mathrm{~g}$, green area) and control gel ( $0.23 \mathrm{~g}$, blue area). The mouse was imaged at 4, 24 and 48 hours through SPECT. 


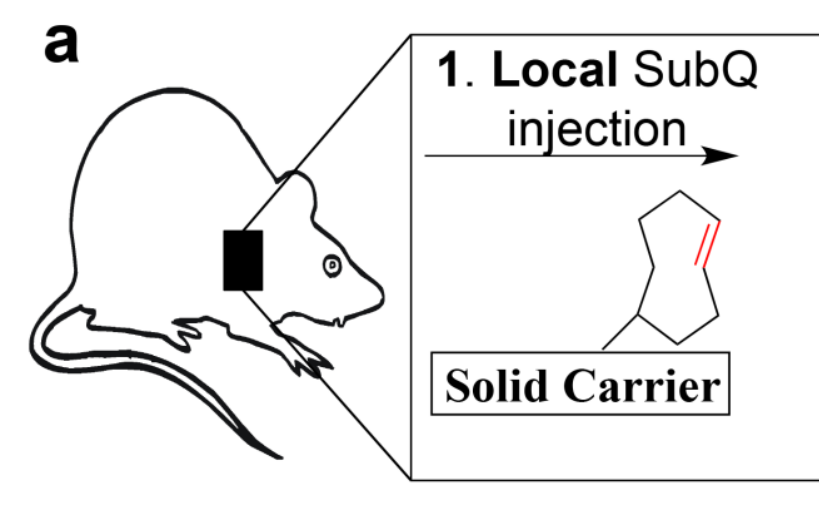

b

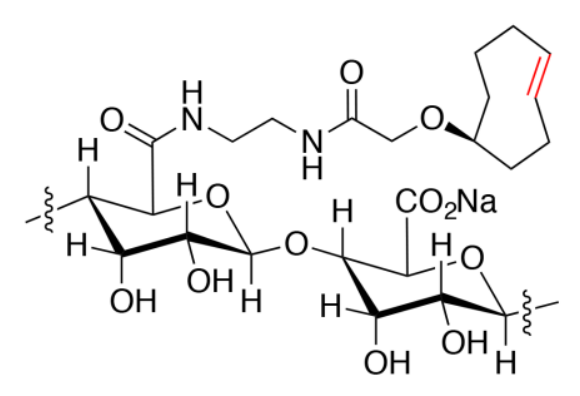

1

trans-cyclooctene modified gel

(TCO-Gel)

2. Systemic IV injection

Therapy

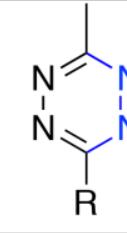

O

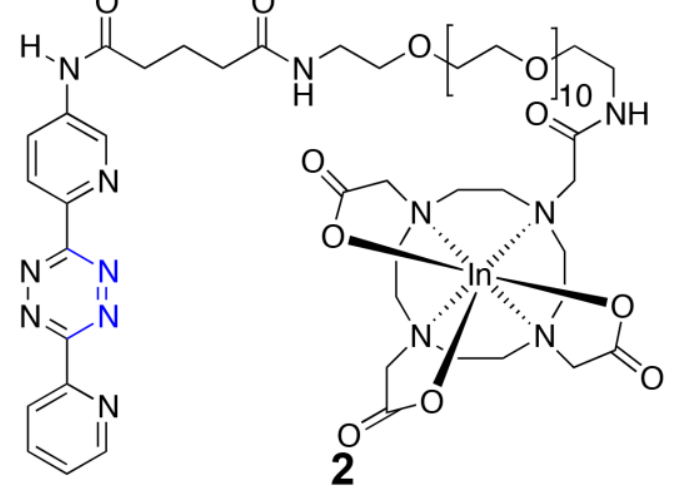

Tetrazine-indium-111

( $T z$ radioprobe) 


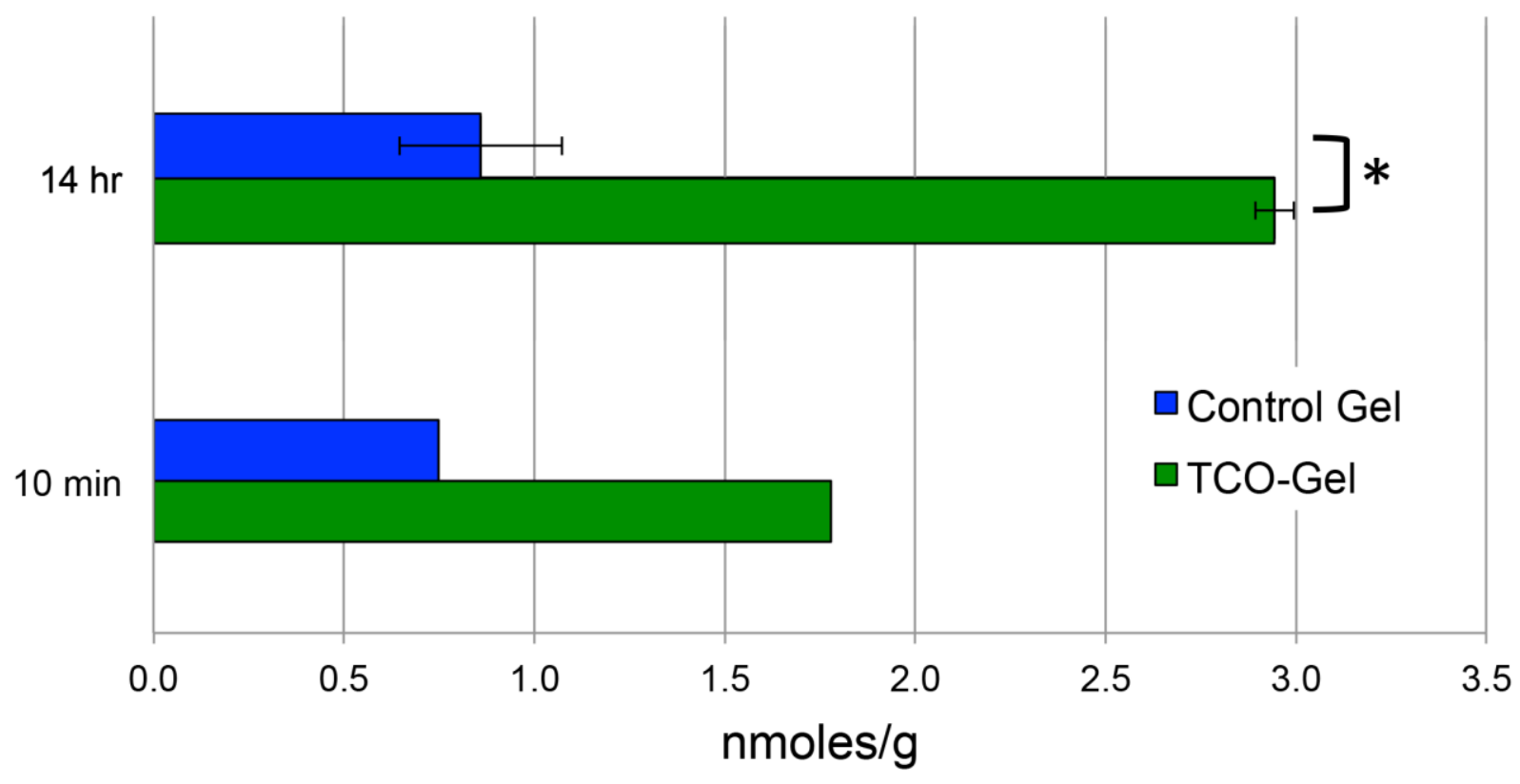




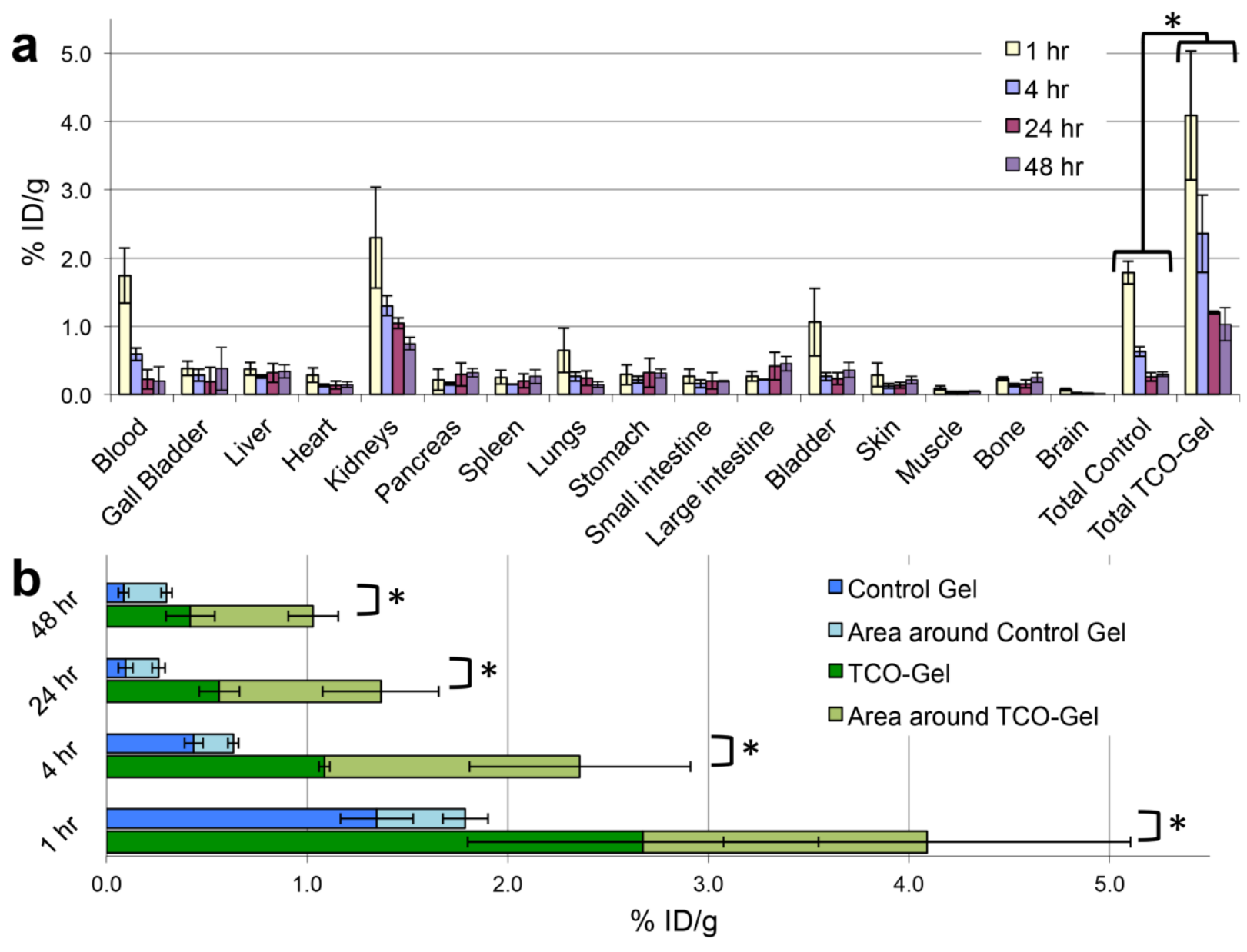




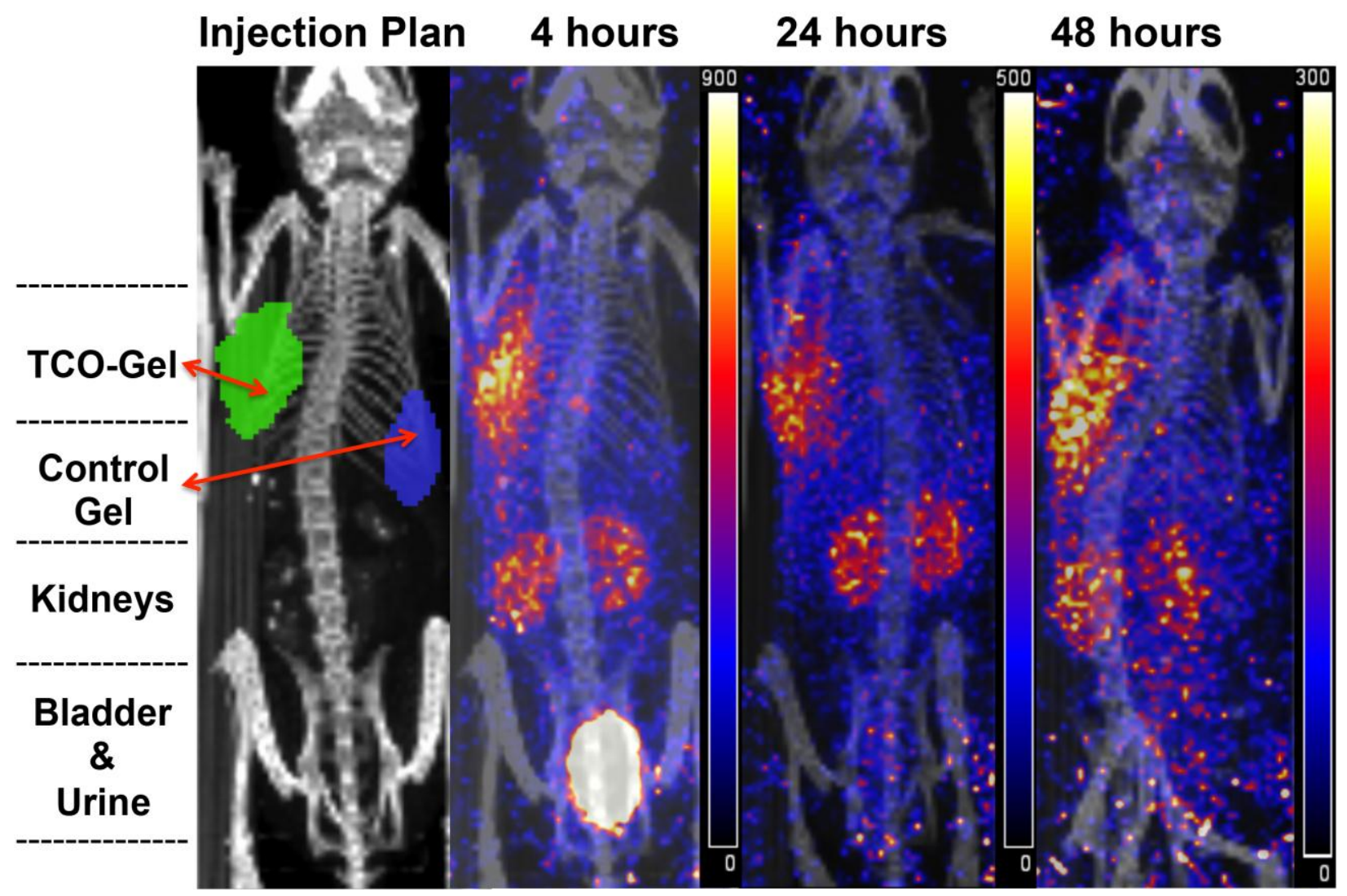




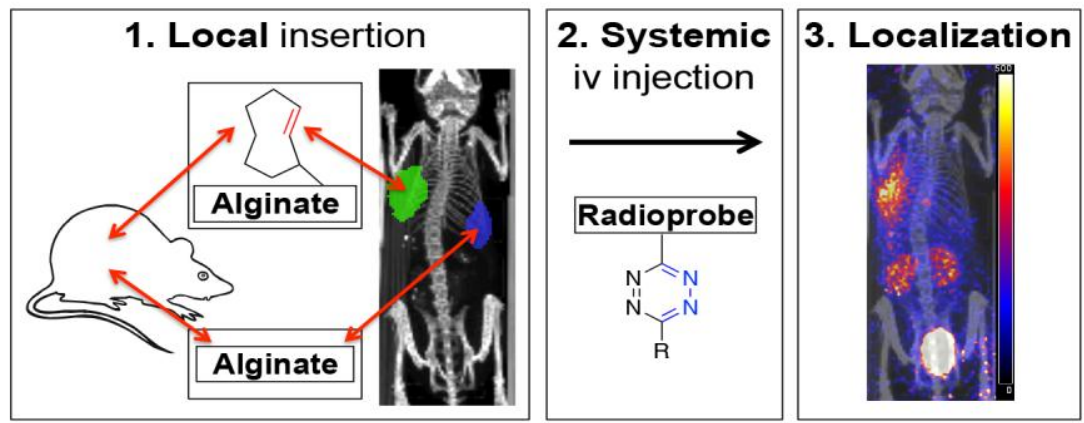

